University of Nebraska - Lincoln DigitalCommons@University of Nebraska - Lincoln

Biological Systems Engineering: Papers and

Publications

Biological Systems Engineering

$5-2013$

\title{
Two-Dimensional Blood Flow Velocity Estimation Using Ultrasound Speckle Pattern Dependence on Scan Direction and A-Line Acquisition Velocity
}

Tiantian Xu

University of Nebraska-Lincoln

Gregory R. Bashford

University of Nebraska-Lincoln, gbashford2@unl.edu

Follow this and additional works at: http://digitalcommons.unl.edu/biosysengfacpub

Part of the Bioresource and Agricultural Engineering Commons, and the Civil and Environmental Engineering Commons

Xu, Tiantian and Bashford, Gregory R., "Two-Dimensional Blood Flow Velocity Estimation Using Ultrasound Speckle Pattern Dependence on Scan Direction and A-Line Acquisition Velocity" (2013). Biological Systems Engineering: Papers and Publications. 382. http://digitalcommons.unl.edu/biosysengfacpub/382

This Article is brought to you for free and open access by the Biological Systems Engineering at DigitalCommons@University of Nebraska - Lincoln. It has been accepted for inclusion in Biological Systems Engineering: Papers and Publications by an authorized administrator of DigitalCommons@University of Nebraska - Lincoln. 


\title{
Two-Dimensional Blood Flow Velocity Estimation Using Ultrasound Speckle Pattern Dependence on Scan Direction and A-Line Acquisition Velocity
}

\author{
Tiantian Xu and Gregory R. Bashford
}

\begin{abstract}
We have previously investigated the change of apparent lateral speckle size caused by the direction and spatial rate of scanner A-line acquisition (scan velocity). An algorithm which measures the lateral component of blood flow velocity was developed based on the increase in speckle size resulting from relative motion between moving scatterers and the scan velocity. In this paper, the change of the apparent dominant angle of the speckle pattern in a straight vessel was investigated and a new method of two-dimensional blood flow velocity estimation is introduced. Different scan velocities were used for data acquisition from blood flow traveling at an angle relative to the ultrasound beam. The apparent angle of the speckle pattern changes with different scan velocities because of misregistration between the ultrasound beam and scatterers. The apparent angle of the speckle pattern was resolved by line-to-line cross-correlation in the fast-time (axial) direction on a region-of-interest (ROI) in each blood flow image and used to spatially align the ROI. The resulting lateral speckle size within the aligned ROI was calculated. The lateral component of the blood flow is shown to be closest to the scan velocity which gives the maximum speckle size and the apparent angle of speckle pattern collected by this scan velocity is the best estimate for the actual angle of blood flow. These two components produce two-dimensional blood flow velocity estimations. This method was studied through both computer simulation and experiments with a blood flow phantom. Nine scan velocities were used to collect blood flow data with velocities ranging from 33 to $98 \mathrm{~cm} / \mathrm{s}$ and four beam-to-flow angles. In simulated plug blood flow, the mean bias of angle estimation is below $2 \%$ with an average standard deviation of $3.6 \%$. In simulated parabolic blood flow, the angle of blood flow is overestimated because of speckle decorrelation caused by flow gradients and the estimation bias increases with decreasing beam-to-flow angle, which has an average value of $8.8 \%$ and standard deviation of $10 \%$. Because of the complexity of flow profiles in the blood flow phantom, the angle of blood flow is also overestimated and the mean bias is increased by a factor of two compared with simulated parabolic flow. For the velocity estimation results, the mean bias is below $5 \%$ with an average standard deviation of $4.6 \%$ in the simulated plug blood flow. In the simulated parabolic flow and blood flow phantom, the velocity is underestimated because of speckle decorrelation. The mean bias of velocity estimation in the simulated parabolic flow is $-6 \%$ with an average standard deviation of $11.2 \%$. In the blood flow phantom, the mean bias of the velocity estimation is $-5 \%$ with a higher average standard deviation of $21.5 \%$. This method can resolve the angle and amplitude of two-dimensional blood flow simultaneously. The accuracy of
\end{abstract}

Manuscript received September 11, 2012; accepted January 13, 2013. The authors are with the Department of Biological Systems Engineering, University of Nebraska-Lincoln, Lincoln, NE (e-mail: gbashford2@ unl.edu).

DOI http://dx.doi.org/10.1109/TUFFC.2013.2647 the estimation can be further improved by using more scanning velocities.

\section{INTRODUCTION}

$\mathrm{B}$ LOOD flow measurement is one of the major functions of medical ultrasound imaging systems. To measure the velocity of blood flow noninvasively, an ultrasound beam is emitted by an ultrasound transducer into the human body. Ultrasound waves will then be reflected back to the transducer by the scatterers in the blood (typically red blood cells). This process is continuously repeated several times. Based on the location of the blood flow, the reflected signals can be gated at a certain depth. The signal constituted by samples from successive received A-lines (the slow-time signal) contains frequency components proportional to the blood velocity along the ultrasound beam [1]. The frequency distribution can then be calculated by spectral estimation of the sampled signal. Using the Doppler equation, the velocity distribution at the sampling depth can be converted from the frequency spectrum, which is known as spectral Doppler. Instead of emitting the ultrasound beam in one direction, color Doppler performs data acquisition similar to spectral Doppler, but with a much smaller number of samples, in different directions and then estimates the velocity at different depths. The velocity can be found through an autocorrelation approach developed by Kasai et al. [2] and Hein and O'Brien [3]. A color-coded image of velocity is then superimposed on the B-mode image. Although these two Doppler based methods have been widely investigated and commercially implemented, a limitation of these velocity estimation techniques is that only a 1-D projection of the blood velocity vector along the axial dimension of the ultrasound beam can be found, because there is no Doppler frequency shift (actually sample-to-sample phase change) when the transducer face is aligned parallel to the blood flow. Because complex fields are present throughout the arterial system, multidimensional flow imaging is desirable. This would allow better assessment of the flow field and its associated hemodynamic parameters, and hence potentially improve cardiovascular risk assessment [4].

Several 2-D estimation algorithms have been developed for resolving complex flow patterns by estimating both the axial and lateral velocity component. Vector Doppler 
extends 1-D Doppler techniques by emitting ultrasound beams along different angles and making vector velocity estimation through triangulation [5]-[8]. Speckle tracking measures multidimensional blood flow by tracking displacement of the speckle pattern generated by scatterers (typically red blood cells) in blood [9], [10]. Feature tracking simplified speckle tracking by only tracking features of the speckle patterns [11], [12]. Transverse spatial modulation applies lateral modulation to influence the received signal by transverse motion [13], [14]. Other techniques such as multidimensional spectrum analysis [15] and maximum likelihood blood velocity estimation [16] have also been proposed. A more complete review can be found in [17] and [18].

Our previous studies introduced a new way to measure the lateral component of blood flow using the change of apparent speckle size resulting from the direction and spatial rate of scanner A-line acquisition [19]. In this paper, instead of resolving the flow purely lateral to the ultrasound beam, a new method of two-dimensional blood flow velocity measurement based on the apparent angle of the speckle pattern is proposed. The scanning geometry is similar to that used in our previous studies [19]. Nine scan velocities were used in this paper. By comparing the speckle size of aligned blood flow images collected by different scan velocities, the amplitude and direction of the blood flow can then be resolved simultaneously. Phantom experiments and computer simulations were investigated to test the blood flow angle and velocity amplitude estimation performance. The purpose of this paper is to introduce a new method to measure 2-D blood flow velocity using observations of the change in speckle patterns related to scan velocity.

It should be noted that several important parameters could be studied but are not studied here; they will be investigated at a later time. For example, we assume point spread function (PSF) spatial invariance, although all flow algorithms are affected by depth dependence of the impulse response. Elevational (out-of-plane) flow is not considered here (however, although speckle decorrelation would certainly increase, theoretically no speckle elongation effects would occur, resulting in a (correct) zero estimate of flow in the scan plane). Finally, algorithm optimization, such as the effects of optimal ROI selection and signal-to-noise ratio, and hardware/software costs are outside the scope of this paper, although they will certainly be studied in the future.

\section{BACKGROUND}

In our previous studies [19], the flow purely lateral to the ultrasound beam was collected by scanning in the same direction as the blood flow. A similar scanning geometry was used in this paper to collect data from the blood flow with a specific angle relative to the ultrasound beam axis. Different scan velocities were used for data acquisi-

\section{US-Bmode Image of Blood Flow Phantom}

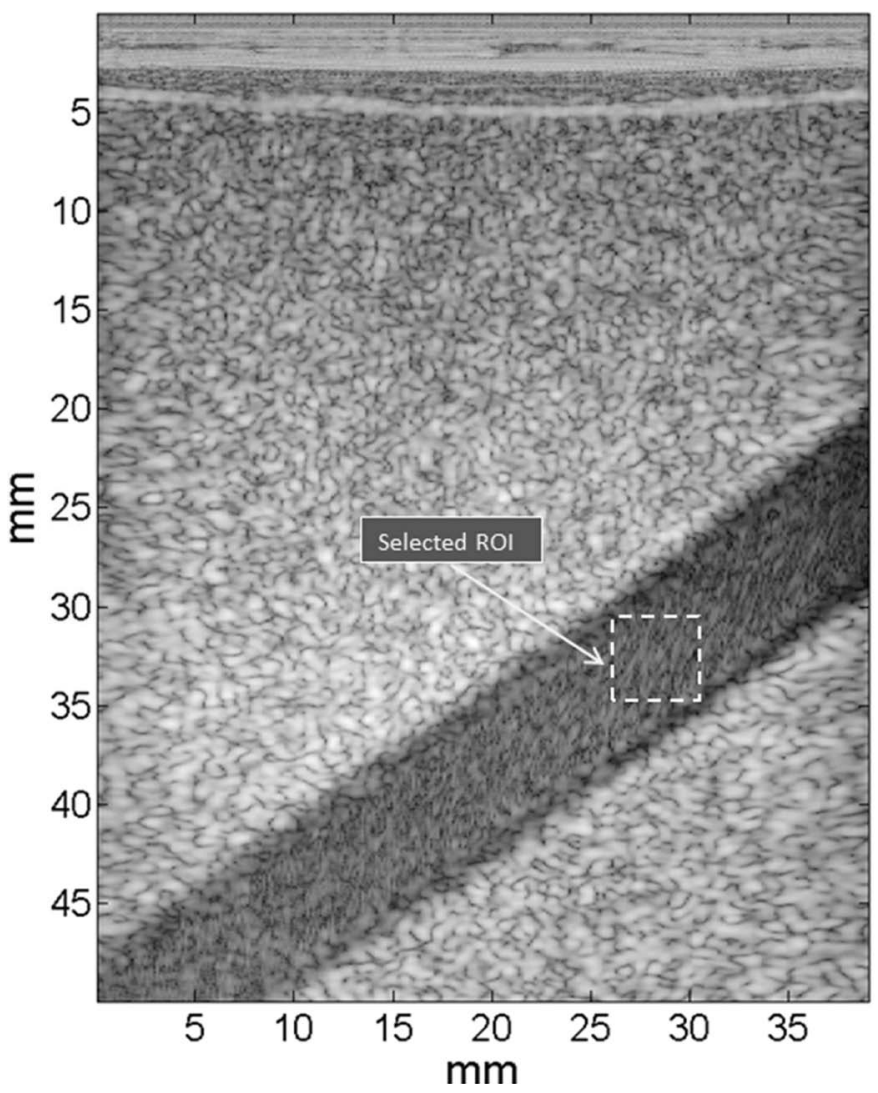

Fig. 1. A $3 \times 4.2 \mathrm{~mm}$ region-of-interest (ROI) is selected from every ultrasound B-mode image of the blood flow phantom.

tion. An ROI was selected from each B-mode image, an example of which can be seen in Fig. 1. Line-by-line crosscorrelation in the fast-time (axial) direction was used to resolve the apparent angle of the speckle pattern, which can be described as follows:

$$
R_{x y}(s)= \begin{cases}\sum_{n=0}^{N-s-1} x_{n+s} y_{n}^{*} & s \geq 0 \\ R_{y x}^{*} & s<0,\end{cases}
$$

where $x$ and $y$ represent two successive A-lines (adjacent scan lines in both time and space) from the ROI and $s$ is the lag between these two A-lines. The cross-correlation coefficient was calculated for each $s$, and the value $s$ which gives the maximum cross-correlation coefficient between $x$ and $y$ was recorded as their distance shift $d_{x y}$. This process was done for every pair of successive A-lines in the ROI and the $d_{x y}$ with the highest frequency of occurrence (i.e., the mode) was considered to be the overall distance shift of the ROI, $d_{\mathrm{ROI}}$. Given the line increment of scanning, $d_{\text {incre }}$, the apparent angle $\theta_{\mathrm{a}}$ of the speckle pattern was calculated as

$$
\theta_{\mathrm{a}}=\tan ^{-1}\left(\frac{d_{\text {incre }}}{d_{\text {ROI }}}\right)
$$


As described previously, the apparent angle of speckle pattern is estimated by line-to-line cross-correlation in the fast-time (axial) direction on the speckle pattern of the blood flow images. However, our experiments showed that the apparent angle $\theta_{\mathrm{a}}$ is not always the same as the actual blood flow angle $\theta$. They only equal each other when the lateral component of blood flow is the same as scan velocity. The relationship between these two angles can be shown as

$$
\begin{cases}\theta_{\mathrm{a}}<\theta & \left(V_{\text {lateral }}>V_{\text {scan }}\right) \\ \theta_{\mathrm{a}}=\theta & \left(V_{\text {lateral }}=V_{\text {scan }}\right) \\ \theta_{\mathrm{a}}>\theta & \left(V_{\text {lateral }}<V_{\text {scan }}\right) .\end{cases}
$$

The reason for this phenomenon is visually demonstrated in Fig. 2, depicting a single scatterer moving from left to right across an ROI in which successive A-lines are also being collected; $\theta$ is the actual angle of blood flow, and $\theta_{\mathrm{a}}$ is the apparent angle of the blood flow. When the lateral component of blood flow is slower than the scan velocity, as is the case in Fig. 2, the ultrasound beams are collecting data laterally faster than the scatterer is moving. Because of the width of the ultrasound PSF, the transducer receives a reflected ultrasound echo even if the scatterer is not precisely at the focal position of each beam. The correct depth information of the scatterers is more accurately preserved as a result of dynamic receive focusing; however, errors will occur in the lateral positions of this scatterer. In Fig. 2, because the lateral component of blood flow velocity is less than the scan velocity, the apparent lateral position of the scatterer will be shifted to the right. As shown by the circles, these positions will be interpreted as the scatterer positions by the transducer. A similar, accumulated effect occurs with many scatterers in the ROI. As a result, the apparent angle of the speckle pattern will be greater than the actual blood flow angle. The opposite phenomenon will occur when the lateral component of blood flow velocity is greater than the scan velocity. Under this condition, the apparent angle of the speckle pattern will be less than the actual blood flow angle.

As discussed in our previous studies [20], the apparent lateral speckle size increases when the scan velocity is close to the lateral component of blood flow because the lateral rate of ultrasound beam movement becomes synchronized with the movement of scatterers. To compare the apparent speckle size of blood flow images collected by different scan velocities, the ROI is first aligned by the apparent angle of the speckle pattern, which means shifting each A-line by $d_{\mathrm{ROI}}$ to make the speckle pattern in the ROI purely lateral, as shown in Fig. 3. Then, the speckle size is calculated by the full-width at half-maximum (FWHM) of the autocovariance function (ACVF) of the ROI [21]. The speckle size will change for different scan velocities. When the speckle size reaches its maximum value, the lateral component of blood flow equals the scan velocity and the apparent speckle angle equals the actual blood flow angle. Thus, in this method, two-dimensional blood flow velocity estimates are made.

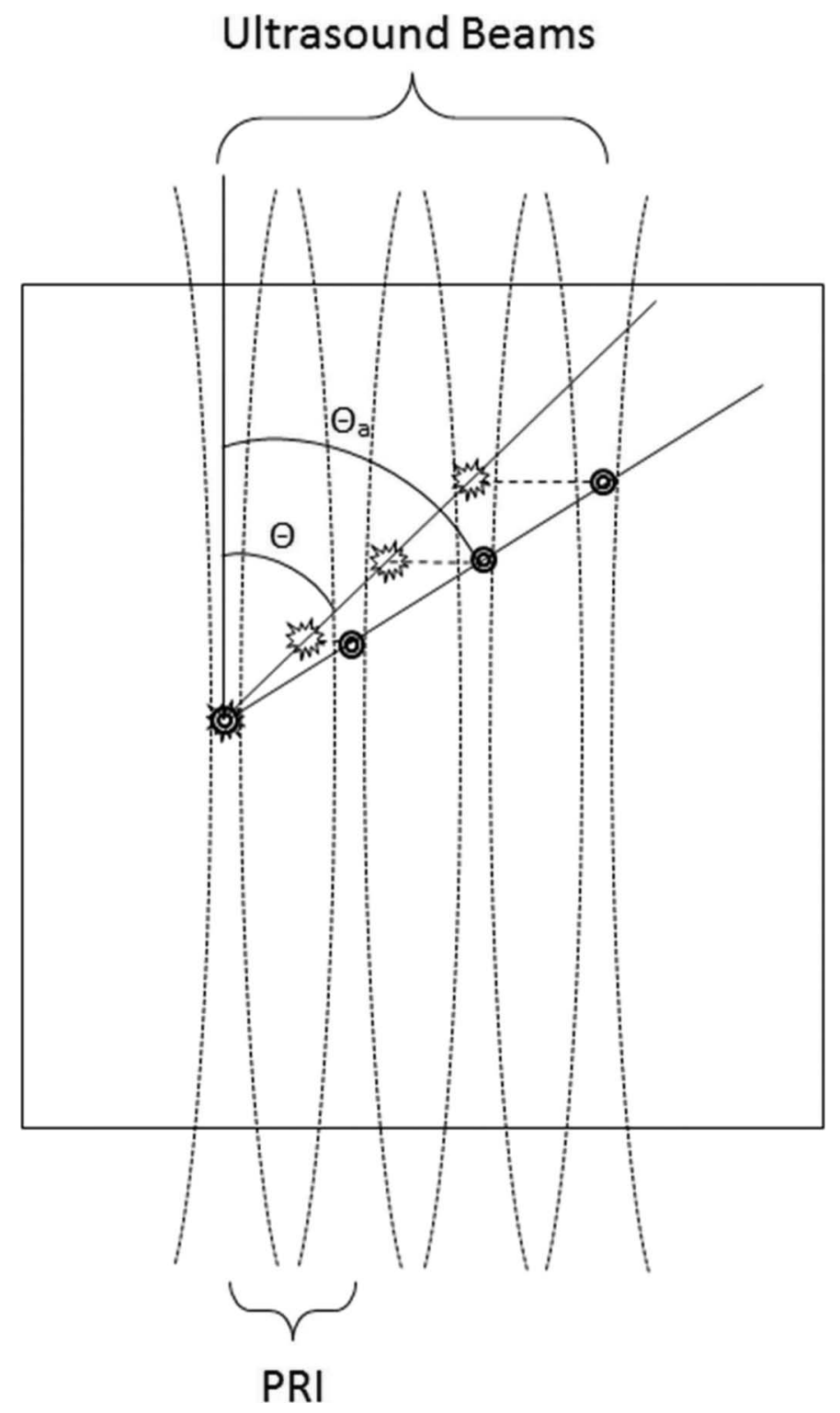

Fig. 2. Representation of the difference between apparent blood flow angle and actual blood flow angle. Burst markers show actual position of an individual scatterer, and circles show where the scatterer is interpreted as existing in the space.

\section{MAterials AND MethodS}

A commercial flow phantom (Optimizer RMI 1425, Gammex, Middleton, WI), was used to simulate blood with an approximately parabolic flow profile. Bloodmimicking fluid which has acoustic properties similar to blood (speed of sound $1550 \mathrm{~m} / \mathrm{s}$, density $1.03 \mathrm{~g} / \mathrm{mL}$ ) was pumped through a tube $(5 \mathrm{~mm}$ inside diameter, $1.25 \mathrm{~mm}$ thickness, $40^{\circ}$ from horizontal) in the phantom, and the tube was surrounded by tissue-mimicking material (speed of sound $1540 \mathrm{~m} / \mathrm{s}$, attenuation $0.5 \mathrm{~dB} / \mathrm{cm} / \mathrm{MHz}$ ). A Sonoline Antares ultrasound imaging system (Siemens Medical Solutions, Issaquah, WA) was used for data acquisition. The VF7-3 linear array transducer (192 elements, $3.33 \mathrm{MHz}$ center frequency) was set to focus on the tube located in the blood flow phantom, with a total imaging 


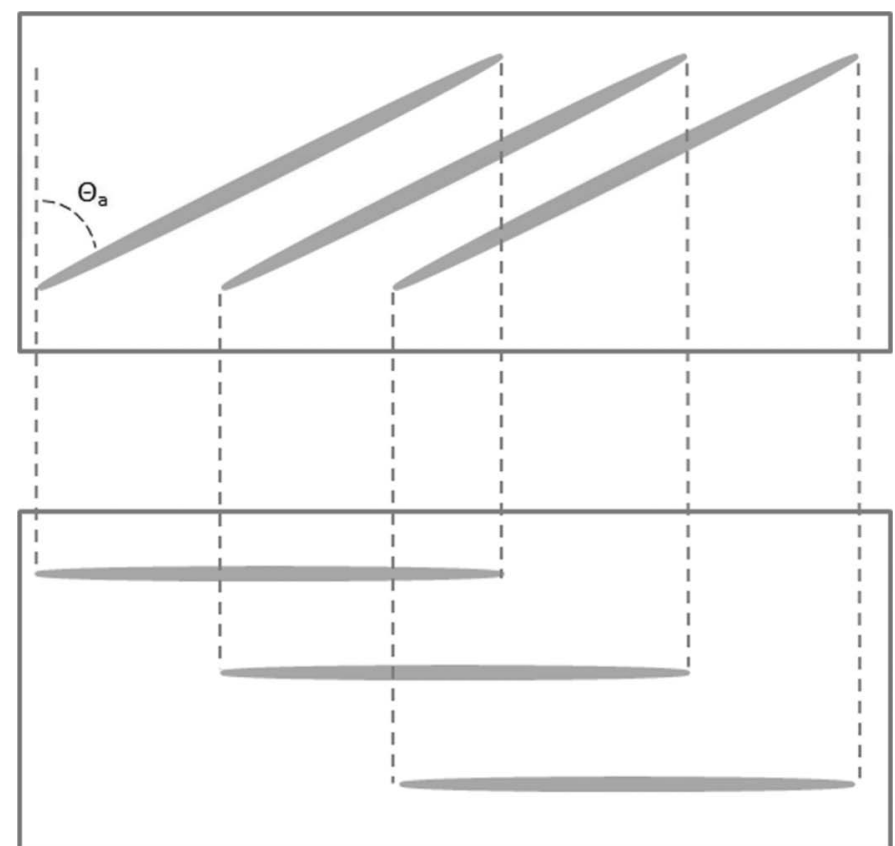

Fig. 3. Each region-of-interest is aligned by cross-correlation and the apparent flow angle $\theta_{a}$ is calculated during alignment.

depth of $5 \mathrm{~cm}$. The Axius Direct ultrasound research interface (URI) was employed to transfer ultrasound data (post-beamformation but before any downstream processing) to a computer for further analysis. Imaging parameters can be accessed from the header information given by the URI, including frame rate, number of A-lines, and beam spacing. Thus, the scan velocity can be calculated given the values of these parameters. In our experiments, nine scan velocities were used: $22,26,31,37,46,52,63$, 75 and $91 \mathrm{~cm} / \mathrm{s}$. Six different flow velocities were set for the flow phantom: $33,41,55,65,80$, and $98 \mathrm{~cm} / \mathrm{s}$. Two angles between the blood flow and the ultrasound beam were used: $50^{\circ}$ and $70^{\circ}$.

A Field II simulation [22], [23] was also used to generate blood flow data for the experiments. At least ten scatterers were assigned to each resolution cell over a 3-D volume to produce a fully developed scatterer field. Four angles between the blood flow and the ultrasound beam were simulated: $10^{\circ}, 30^{\circ}, 50^{\circ}$, and $70^{\circ}$. Other parameters used in the simulation are similar to the Sonoline Antares ultrasound imaging system and the flow phantom as mentioned previously; see Table I. Two flow conditions were simulated: the first flow condition had a plug flow distribution in the vessel. The second flow condition had a parabolic velocity distribution in the flow with maximum velocity at the center and decreasing to zero at the boundary of the vessel, producing a lateral gradient similar to the flow velocity in the blood flow phantom.

Ten images were simulated or collected for each scan. In each image, an ROI with an axial length of $3 \mathrm{~mm}$ and a lateral length of $4.2 \mathrm{~mm}$ was selected from the tube, as shown in Fig. 1. This ROI was then used to calculate the apparent dominant angle of the speckle pattern. After ROI alignment by the dominant angle of the speckle pat- tern, the speckle size of the blood flow images collected by different scan velocities was then calculated to resolve the lateral component and actual angle of the blood flow.

In both simulation and phantom experiments, the actual velocity was taken to be the peak velocity from calculated flow (simulation) or pulsed-wave (PW) Doppler measurement on the Antares (phantom).

\section{RESUlts}

Fig. 4 shows the apparent angle of the speckle pattern and the speckle size of aligned blood flow images when the angle between blood flow and ultrasound beam axis is $50^{\circ}$. Fig. 4(a) shows the apparent angle of the speckle pattern of simulated plug flow collected by different scan velocities. Fig. 4(b) shows the apparent angle of the speckle pattern of simulated parabolic flow collected by different scan velocities, and Fig. 4(c) shows the apparent angle of the speckle pattern of the blood flow phantom collected by different scan velocities. It can be seen that the apparent angle of the speckle pattern is less than the actual blood flow angle when the scan velocity is less than the lateral component of blood flow and greater than the actual blood flow angle when the scan velocity is greater than the lateral component of blood flow. When the scan velocity equals the lateral component of blood flow, the apparent angle of the speckle pattern equals the actual angle of blood flow.

Fig. 4(d) shows the speckle size of the aligned blood flow image of simulated plug flow, Fig. 4(e) shows the speckle size of the aligned blood flow image of simulated parabolic flow, and Fig. 4(f) shows the speckle size of the aligned blood flow image of the blood flow phantom. It can be seen that the speckle size of aligned blood flow is changing with different scan velocities and it reaches the maximum value when the scan velocity equals the lateral component of blood flow.

Similar results on blood flow with an angle of $70^{\circ}$ relative to the ultrasound beam axis are shown in Fig. 5. The relationship between the apparent angle of the speckle

TABle I. Parameters of Transducer and Blood Flow Phantom Used in the Simulation.

\begin{tabular}{ll}
\hline Transducer & $3.33 \mathrm{MHz}$ \\
Center frequency & $7.5 \mathrm{~mm}$ \\
Element height & $0.203 \mathrm{~mm}$ \\
Element width & $25 \mu \mathrm{m}$ \\
Kerf & 192 \\
Number of elements & $40 \mathrm{MHz}$ \\
Sampling frequency & \\
Blood flow phantom & $1550 \mathrm{~m} / \mathrm{s}$ \\
Speed of sound & $50^{\circ}$ relative to the \\
Angle of vessel & ultrasound beam axis \\
& $5 \mathrm{~mm}$ \\
Diameter of vessel & $33,41,55,65,80$, \\
Blood flow velocity & and $98 \mathrm{~cm} / \mathrm{s}$ \\
\hline
\end{tabular}




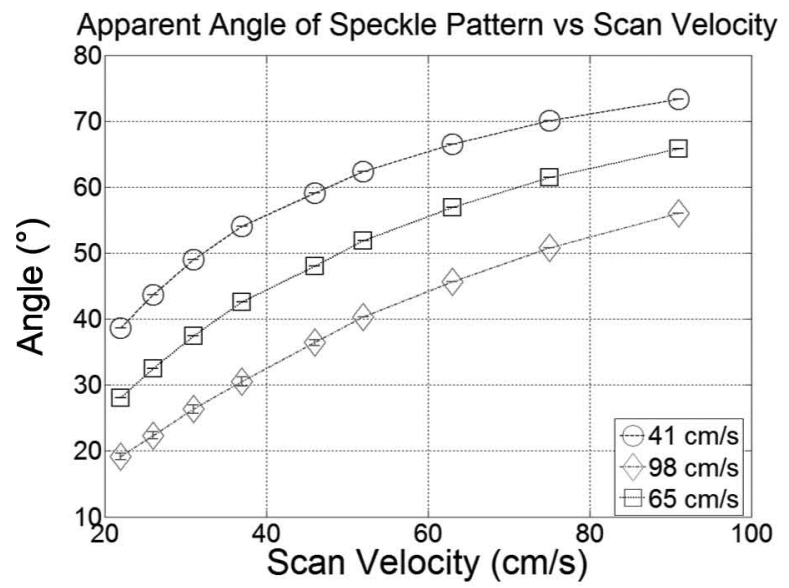

(a)

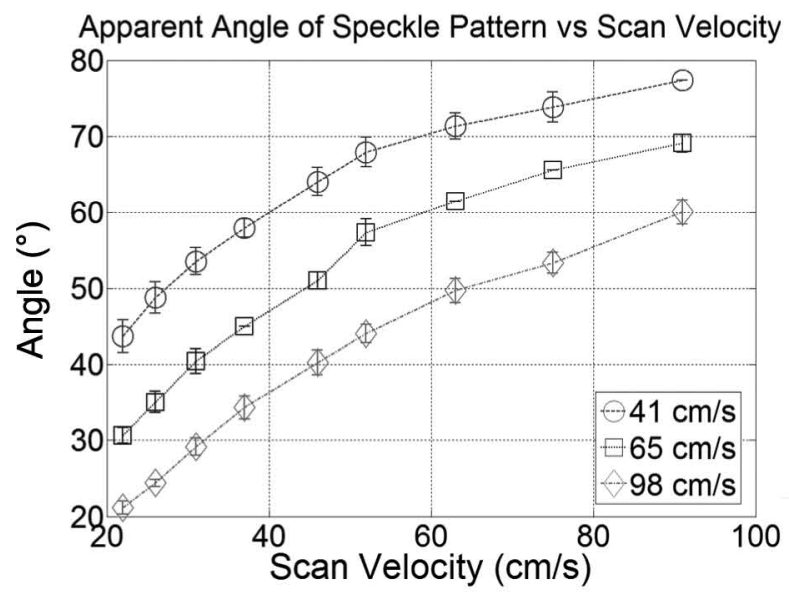

(b)

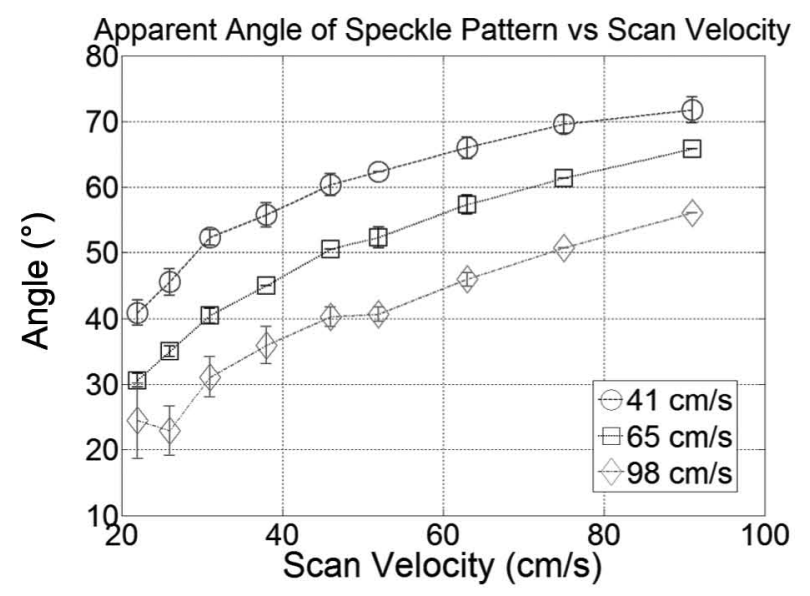

(c)

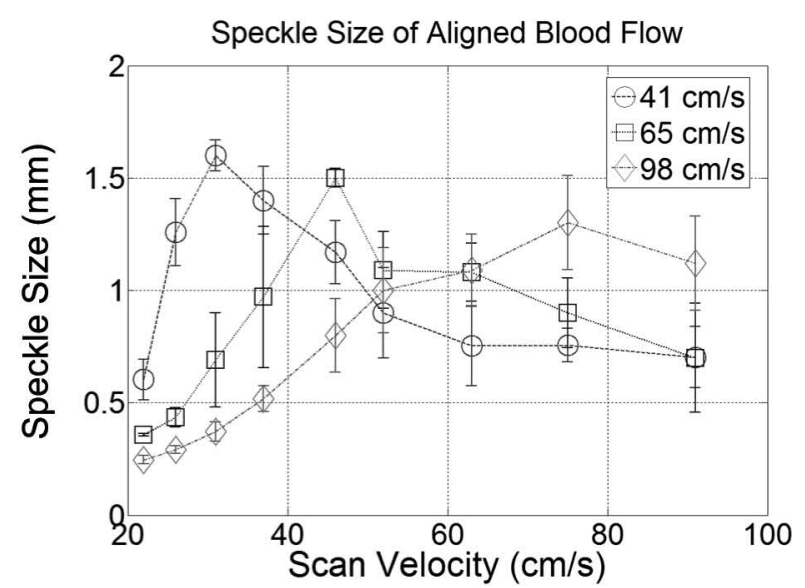

(d)

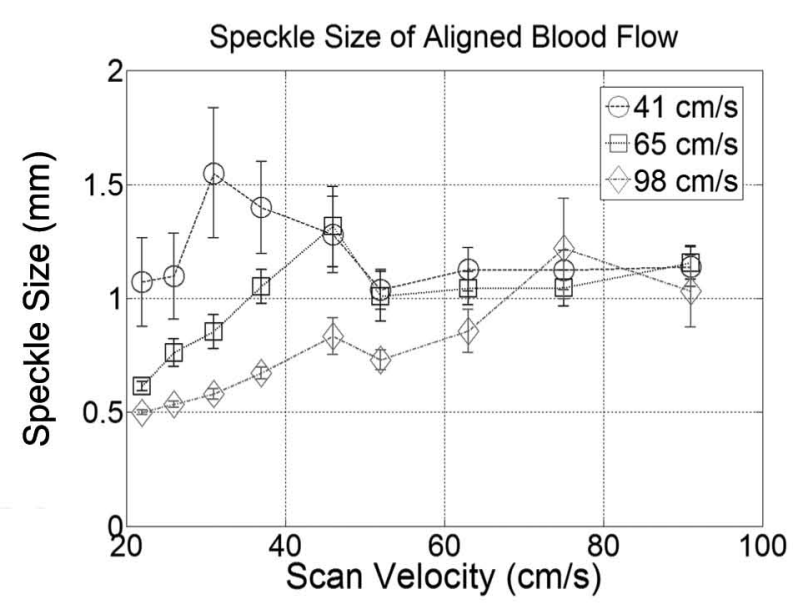

(e)

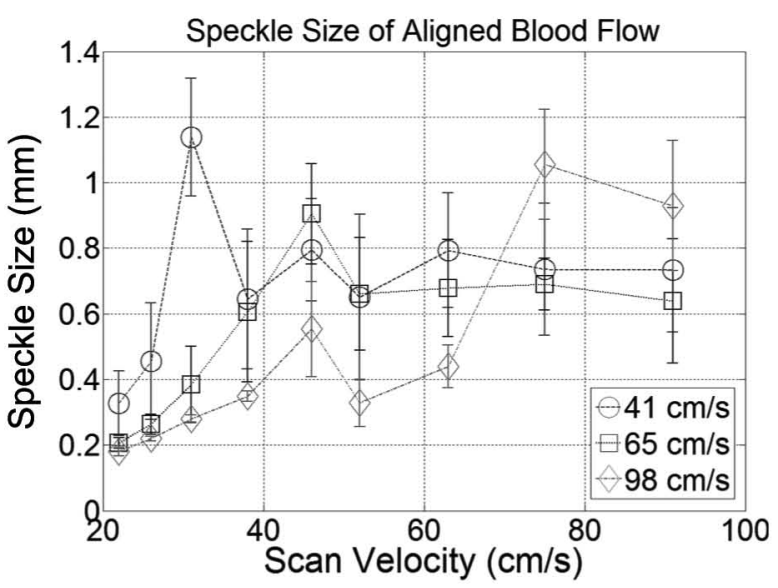

(f)

Fig. 4. The apparent angle of the speckle pattern of the blood flow collected by different scan velocities $(41,65$, and $98 \mathrm{~cm} / \mathrm{s}$ magnitude, 31,50 , and $75 \mathrm{~cm} / \mathrm{s}$ lateral component, $50^{\circ}$ relative to the ultrasound beam) for (a) simulated plug flow, (b) simulated parabolic flow, and (c) blood flow phantom. The speckle size of the aligned blood flow image: (d) simulated plug flow, (e) simulated parabolic flow, and (f) blood flow phantom.

pattern and scan velocities is shown in Figs. 5(a), 5(b), and 5(c) for simulated plug flow, simulated parabolic flow, and blood flow phantom, respectively. The relationship between the speckle size of the aligned blood flow image and scan velocities is shown in Figs. 5(d), 5(e), and 5(f) for simulated plug flow, simulated parabolic flow, and blood flow phantom, respectively.

In Figs. 4 and 5, the lateral component of blood flow and actual blood flow angle can be estimated by searching for the peak speckle size of the aligned blood flow 


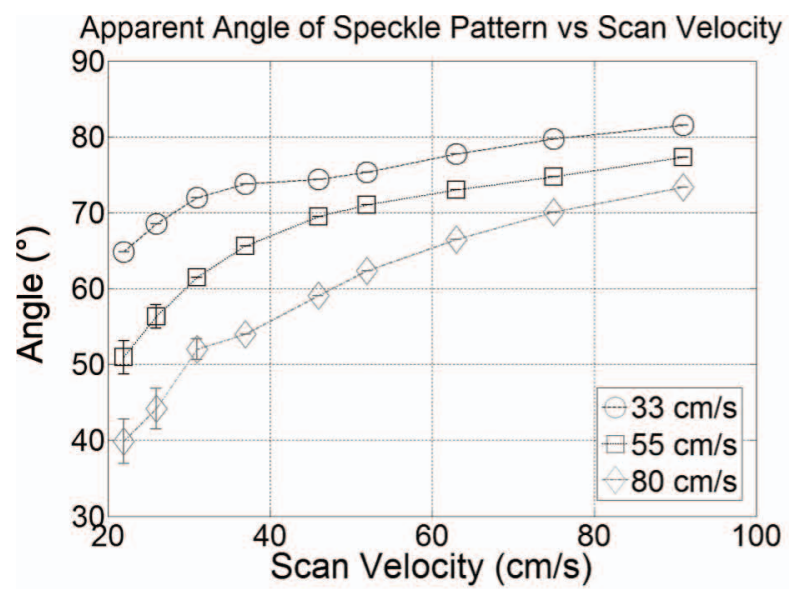

(a)

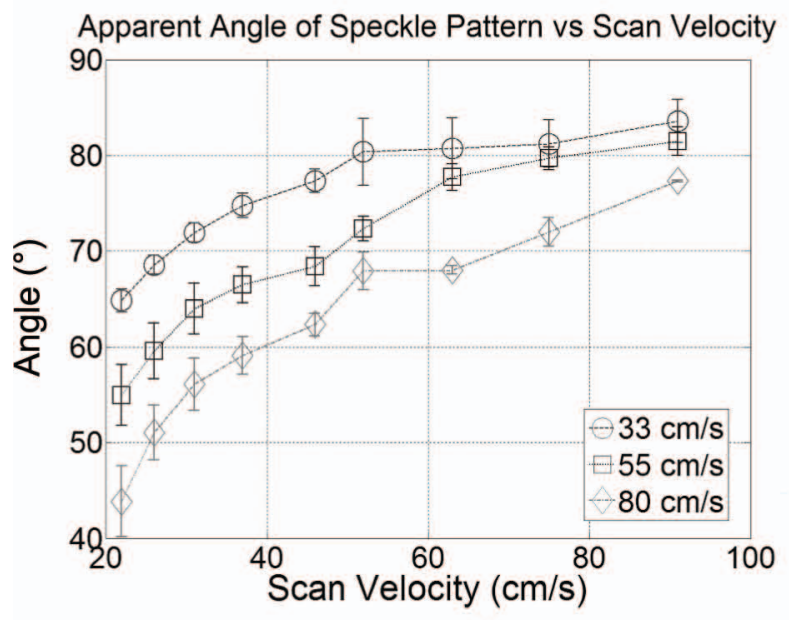

(b)

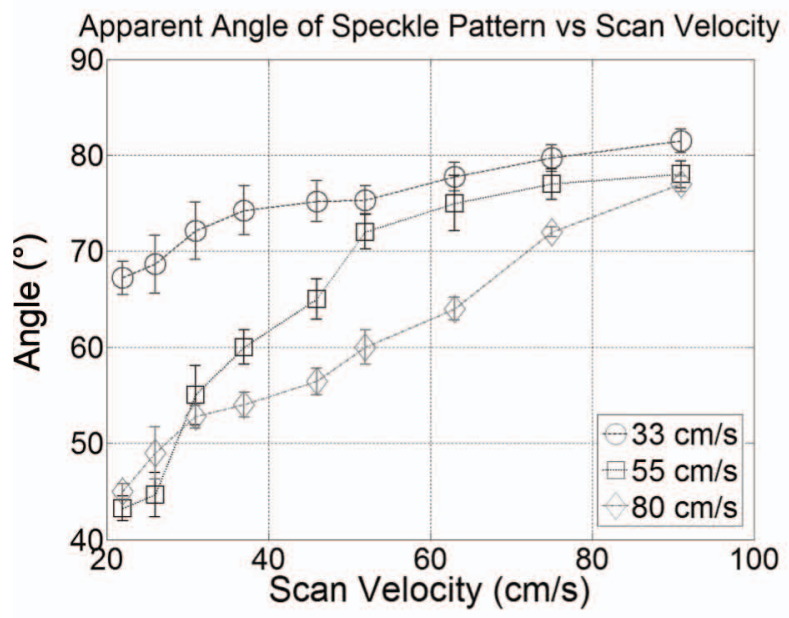

(c)

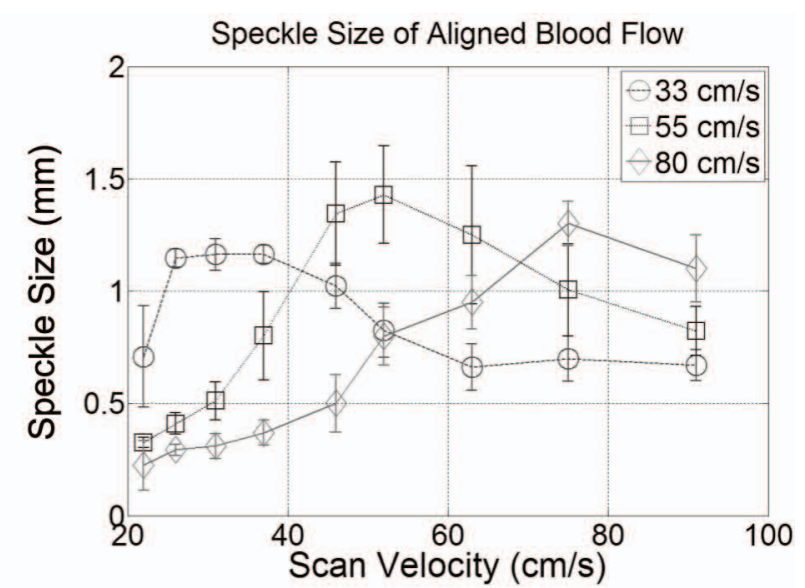

(d)

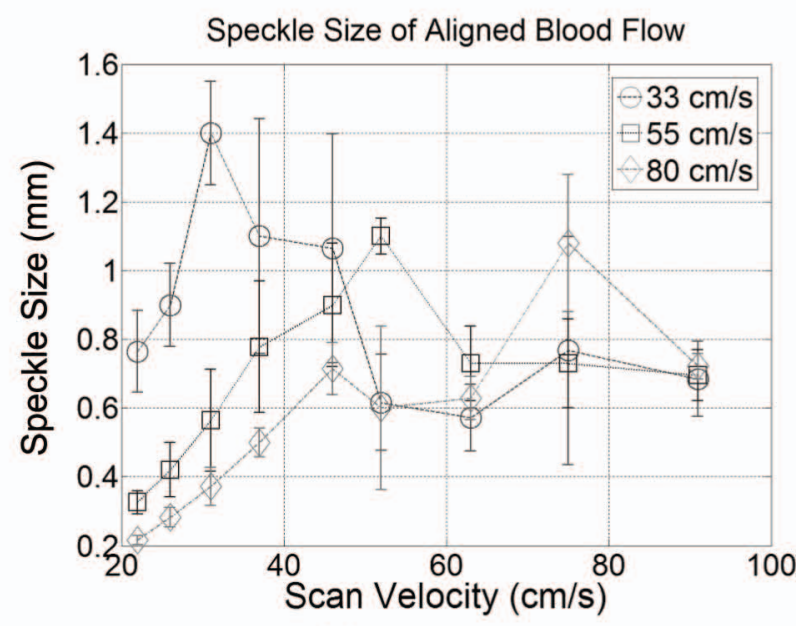

(e)

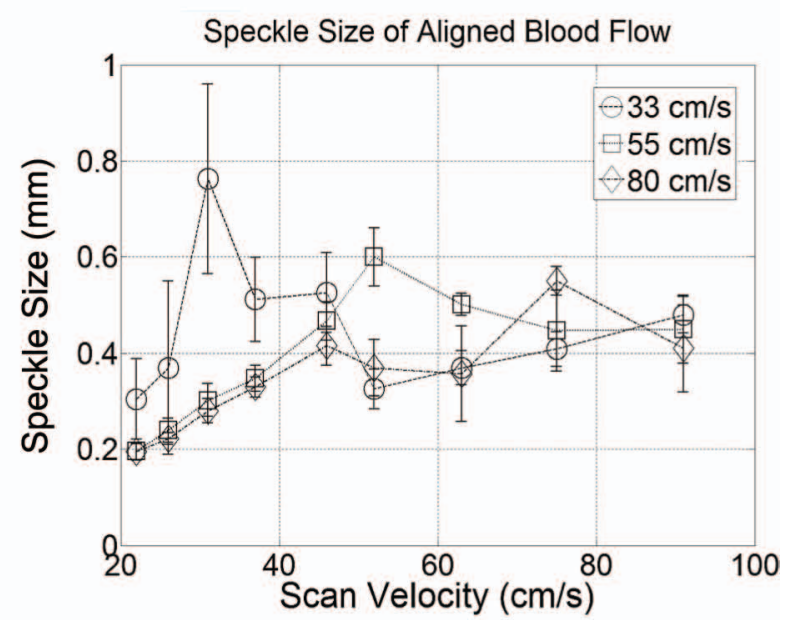

(f)

Fig. 5. The apparent angle of the speckle pattern of the blood flow collected by different scan velocities (33, 55, and 80 cm/s magnitude, 31,52 , and $75 \mathrm{~cm} / \mathrm{s}$ lateral component, $70^{\circ}$ relative to the ultrasound beam) for (a) simulated plug flow, (b) simulated parabolic flow, and (c) blood flow phantom. The speckle size of the aligned blood flow image: (d) simulated plug flow, (e) simulated parabolic flow, and (f) blood flow phantom.

image collected by different scan velocities. Using these results, the estimated velocities were plotted versus actual velocities in Fig. 6. Fig. 6(a) shows the reference line and estimated velocities versus actual velocities in simulated plug flow, Fig. 6(b) shows the reference line and estimated velocities versus actual velocities in simulated parabolic flow, and Fig. 6(c) shows the reference line and estimated velocities versus actual velocities in the blood flow phan- 


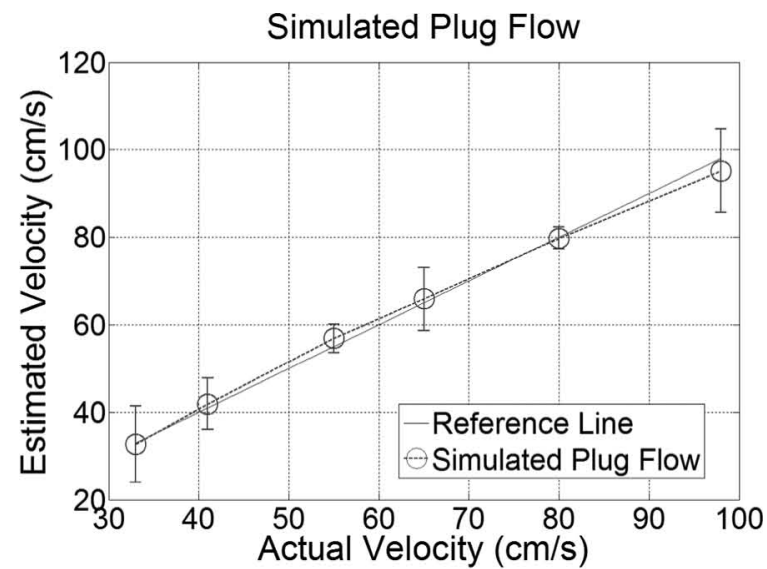

(a)

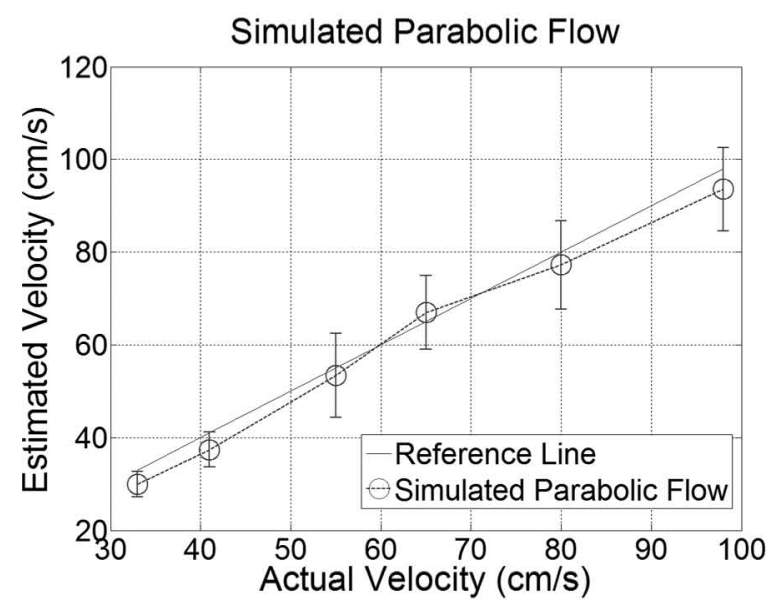

(b)

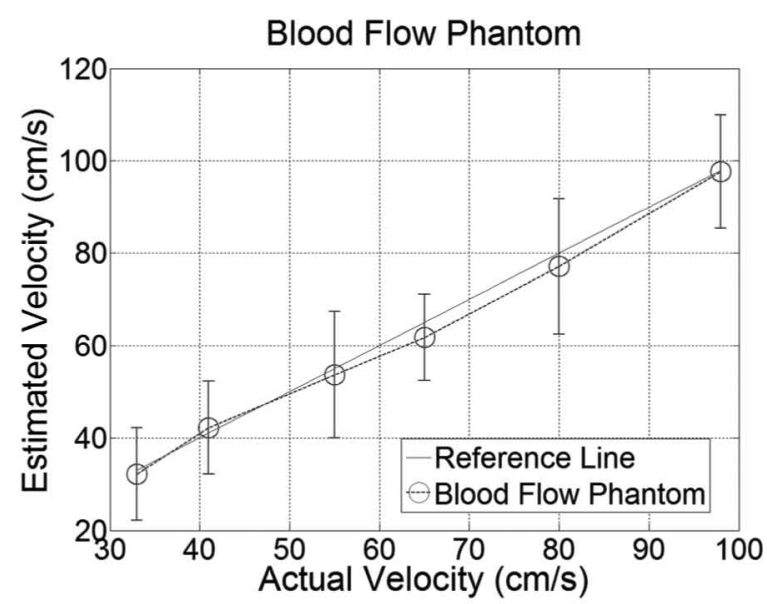

(c)

Fig. 6. Estimated velocities versus actual velocities: (a) simulated plug flow, (b) simulated parabolic flow, and (c) blood flow phantom.

tom. These graphs show the mean and standard deviation of the estimated velocities. Additional results including the mean and standard deviation of estimation bias can be seen in Table II.

Fig. 7 shows the relationship between the estimated blood flow angle and actual blood flow angle. Fig. 7(a) shows the estimated blood flow angle versus actual blood flow angle in the simulated plug flow data, Fig. 7(b) shows the estimated blood flow angle versus actual blood flow angle in the simulated parabolic flow, and Fig. 7(c) shows the estimated blood flow angle versus actual blood flow angle in the blood flow phantom. They were plotted with a reference line to represent the estimation accuracy. Further details of the flow angle estimation bias can be seen in Table III.

\section{Discussion}

The speckle size of the aligned blood flow image changes with different scan velocities, which can be seen in Figs. 4(e) and 4(f) and Figs. 5(e) and 5(f). Generally, the maximum speckle size of aligned blood flow is around $1.5 \mathrm{~mm}$ in the simulated plug flow. In the simulated parabolic flow and blood flow phantom, this value decreased to $1.2 \mathrm{~mm}$ and $1.0 \mathrm{~mm}$ separately. We reason that the flow gradients in the simulated parabolic flow and blood flow phantom cause speckle decorrelation, which decreases the speckle size. Nevertheless, because the speckle pattern will be maximally correlated when the scan velocity equals the lateral component of blood flow, searching for the maximum speckle size of aligned blood flow image can resolve the lateral component of blood flow velocity. In Fig. 4, the maximum speckle size of aligned blood flow image occurs when the scan velocities are 31,52 , and $75 \mathrm{~cm} / \mathrm{s}$. These scan velocities are equal to the lateral component of blood flow with an angle of $50^{\circ}$ relative to the ultrasound beam axis $\left(41 \times \sin 50^{\circ}=31,65 \times \sin 50^{\circ}=50\right.$, and $98 \times \sin 50^{\circ}$ $=75 \mathrm{~cm} / \mathrm{s}$ ). Similar results can be seen in Fig. 5 . When the scan velocities are 31,52 , and $75 \mathrm{~cm} / \mathrm{s}$, the speckle size of the aligned blood flow image reaches its maximum value because these scan velocities equal the lateral component of blood flow with an angle of $70^{\circ}$ relative to the ultrasound beam axis $\left(33 \times \sin 70^{\circ}=31,55 \times \sin 70^{\circ}=\right.$ 52 , and $\left.80 \times \sin 70^{\circ}=75 \mathrm{~cm} / \mathrm{s}\right)$.

The results in Figs. 4(a)-4(c) and Figs. 5(a)-5(c) show that the apparent angle of speckle pattern increases with scan velocity. The reason is the misregistration between the ultrasound beam scan and scatterer movement. When the scan velocities are 31,52 , and $75 \mathrm{~cm} / \mathrm{s}$, which give the maximum speckle size of aligned blood flow image, the apparent angle of speckle pattern equals the actual angle of blood flow, which is $50^{\circ}$ in Fig. 4 and $70^{\circ}$ in Fig. 5 . In the simulated plug flow, the estimation error of blood flow angle is low, which can be seen in Figs. 4(a) and 5(a). In Fig. 4(a), the estimated blood flow angle is $49.2^{\circ}$, $51.3^{\circ}$, and $50.7^{\circ}$ when the angle between the blood flow and ultrasound beam axis is $50^{\circ}$. In Fig. 5(a), the estimated blood flow angle is $71.3^{\circ}, 70.8^{\circ}$, and $70.1^{\circ}$ when the angle between the blood flow and ultrasound beam axis is $70^{\circ}$. However, in simulated parabolic flow and the blood flow phantom, the estimated blood flow angle is generally greater than the actual angle of blood flow. The reason is that the flow gradient causes speckle decorrelation in 
Table II. The Mean and Standard Deviation of Velocity Estimation Bias.

\begin{tabular}{lcccccc}
\hline Simulated plug flow & & & & & \\
Actual velocity (cm/s) & 33 & 41 & 55 & 65 & 80 & 98 \\
Mean bias (\% of actual) & -0.9 & 2.2 & 3.4 & 1.4 & -0.3 & -2.9 \\
SD of bias (\% of actual) & 6.5 & 1.4 & 5.9 & 1.3 & 2.1 & 9.6 \\
Simulated parabolic flow & & & & & & \\
Actual velocity (cm/s) & 33 & 41 & 55 & 65 & 80 & 98 \\
Mean bias (\% of actual) & -12.2 & -7.8 & -8.3 & 3.4 & -5.9 & -4.6 \\
SD of bias (\% of actual) & 8.3 & 9.1 & 16.4 & 12.1 & 11.9 & 9.2 \\
Blood flow phantom & & & & & & 98 \\
Actual velocity (cm/s) & 33 & 41 & 55 & 65 & 80 & -3.5 \\
Mean bias (\% of actual) & -3.4 & 6.1 & -8.5 & -15.1 & -6.8 & 12.5 \\
SD of bias (\% of actual) & 30.5 & 24.7 & 24.9 & 18.3 & 17.3 & 12.9 \\
\hline
\end{tabular}

the axial direction, which will reduce $d_{\mathrm{ROI}}$ during crosscorrelation. Because the apparent angle of speckle pattern is calculated by $(2)$, when $d_{\mathrm{ROI}}$ decreases, the estimated angle of blood flow will be greater than the actual angle of blood flow. This can also be seen in Fig. 7. Fig. 7(a) shows that the estimated angle of simulated plug flow is close to the actual angle with mean bias of less than $2 \%$. However, in the simulated parabolic flow, the estimation bias increases because of the flow gradient. Furthermore, the estimation bias increases with decreasing angle between the blood flow and ultrasound beam axis. We reason that decorrelation in the axial direction caused by the flow gradient in a parabolic flow increases with decreasing angle between blood flow and the ultrasound beam axis. Because the angle of blood flow is estimated by axial cross-correlation on the ROI, when the angle between the blood flow and ultrasound beam axis decreases, the angle estimation bias increases. Fig. 7(c) shows the estimated flow angle versus actual flow angle in the blood flow phantom. Compared with simulated parabolic flow, it can be seen that the estimation bias increases because of the increasing complexity of the flow profile in the blood flow phantom. Details of the angle estimation bias can be seen in Table III. It shows that the mean bias of angle estimation ranges from $0.3 \%$ to $1.5 \%$ with an average value of $0.4 \%$. The standard deviation of bias ranges from $2.1 \%$ to $5.1 \%$ with an average value of $3.6 \%$. In the simulated parabolic flow, because of the flow gradient, the mean bias of angle estimation ranges from $3.2 \%$ to $16.3 \%$ with an average value of $8.8 \%$. The standard deviation of bias ranges from $3.15 \%$ to $23.4 \%$ with an average value of $10.5 \%$. As discussed previously, the estimation bias increases by a factor of six when the angle between the blood flow and the ultrasound beam axis decreases from $70^{\circ}$ to $10^{\circ}$. In the blood flow phantom, the estimation bias is increased by a factor of two compared with simulated parabolic flow because of the complexity of the flow profile in the blood flow phantom.

The relationship between estimated velocities and actual velocities can be seen in Fig. 6. In simulated plug flow, the estimated velocities distribute close to the reference line. However, in the simulated parabolic flow and blood flow phantom, most of the estimated velocities are lower than the actual blood flow velocities; the reason is that axial speckle decorrelation causes overestimation of blood flow angle as discussed previously. Given the lateral component of blood flow velocity, when the angle of blood flow is overestimated, the blood flow velocity will be underestimated. Details of the estimation bias can be seen in Table II. In simulated plug flow, the mean of the estimation bias ranges from $-3 \%$ to $4 \%$, with an average value of $2 \%$ and the standard deviation of the estimation bias ranges from $1.3 \%$ to $9.6 \%$, with an average value of $4.6 \%$. Because of the flow gradient in the simulated parabolic flow, the mean of the estimation bias ranges from $-12.2 \%$ to $3.4 \%$, with an average value of $-5.9 \%$. Most of the estimation bias is negative because the blood flow velocities were underestimated, as discussed previously. The standard deviation of estimation bias also increased, ranging from $8.3 \%$ to $16.4 \%$, with an average value of $11.2 \%$. In the blood flow phantom, the complexity of flow profile causes more speckle decorrelation. As a result, the mean of the estimation bias ranges from $-15.1 \%$ to $3.1 \%$, with an average value of $-5.2 \%$. The standard deviation of estimation bias ranges from $12.5 \%$ to $30.5 \%$, with an average value of $21.5 \%$. Because the underestimation of blood flow velocity is a result of speckle decorrelation, future studies may quantitatively investigate and compensate the underestimation to improve the accuracy of velocity estimation. Furthermore, only nine scan velocities were used in this experiment. In future studies, more scan velocities may be applied by a refined algorithm to increase the estimation accuracy.

Spectrum analysis was also investigated in this experiment. An ROI was selected from the blood flow images, and the 2-D power spectrum of the ROI was calculated and compared for different scan velocities. The results showed that when the scan velocity equals the lateral component of the blood flow, the speckle pattern of the blood flow image was most spatially coherent because the scatterers and ultrasound beam were most correlated in this condition, and the power spectrum of the blood flow image had a high Q-factor (energy is less distributed). When the scan velocity was higher or lower than the lateral component of blood flow, the speckle pattern was less coherent because of misregistration between scatterers and the ultrasound beam. As a result, the power spectrum of the blood flow image was more distributed (lower Q-factor), which can 


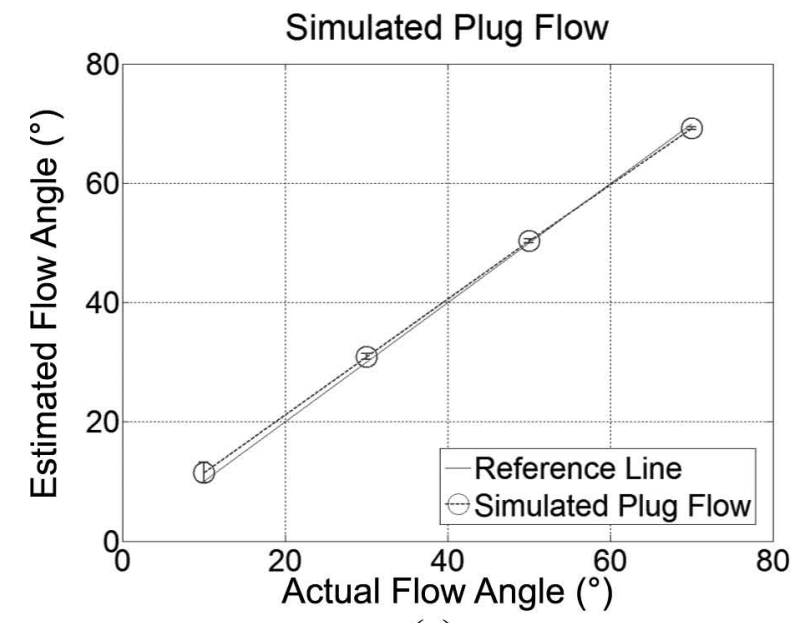

(a)

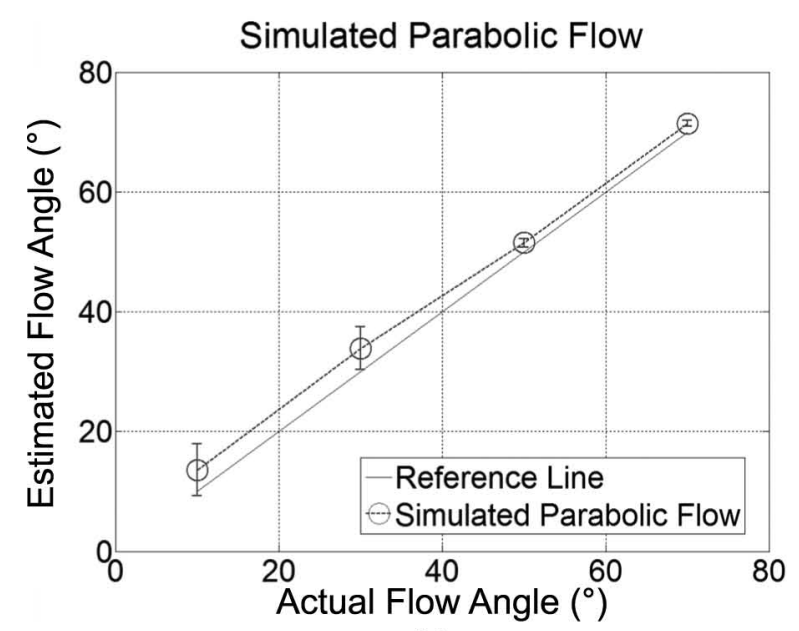

(b)

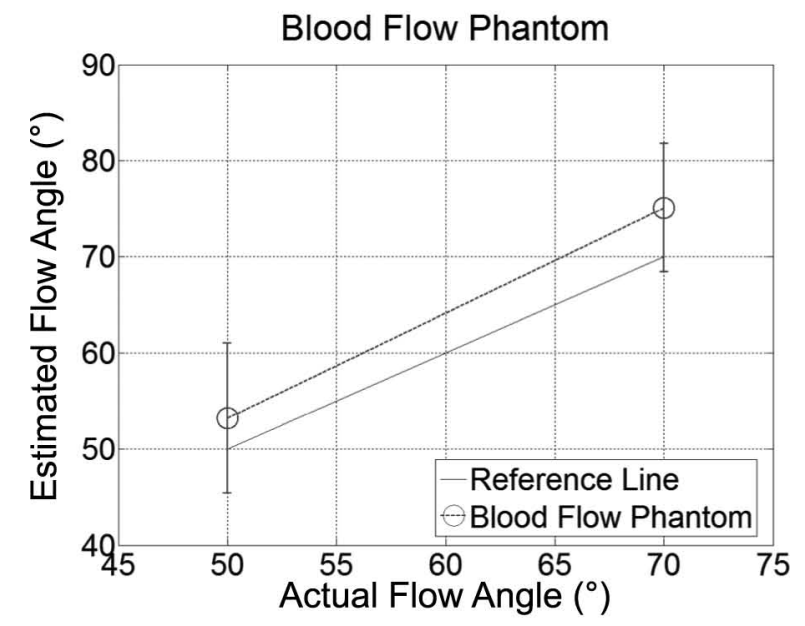

(c)

Fig. 7. Estimated flow angle versus actual flow angle: (a) simulated plug flow, (b) simulated parabolic flow, and (c) blood flow phantom.

be seen in Figs. 8 and 9. Fig. 8 shows the results from Field II simulation where the A-line increment was less than one-fourth of the lateral beam width. It can be seen that the Q-factor of the power spectrum is high when the scan velocity $(50 \mathrm{~cm} / \mathrm{s})$ is close to the lateral component
Table iII. The Mean and Standard Deviation of Angle Estimation Bias.

\begin{tabular}{|c|c|c|c|c|}
\hline \multicolumn{5}{|l|}{ Simulated plug flow } \\
\hline Actual angle $\left(^{\circ}\right)$ & 10 & 30 & 50 & 70 \\
\hline Mean bias (\% of actual) & 1.5 & 0.3 & 0.3 & 0.6 \\
\hline SD of bias ( $\%$ of actual) & 3.2 & 2.1 & 5.1 & 4.1 \\
\hline \multicolumn{5}{|l|}{ Simulated parabolic flow } \\
\hline Actual angle $\left(^{\circ}\right)$ & 10 & 30 & 50 & 70 \\
\hline Mean bias (\% of actual) & 16.3 & 11.2 & 4.5 & 3.2 \\
\hline SD of bias ( $\%$ of actual) & 23.4 & 8.2 & 7.5 & 3.15 \\
\hline \multicolumn{5}{|l|}{ Blood flow phantom } \\
\hline Actual angle $\left({ }^{\circ}\right)$ & \multicolumn{2}{|c|}{50} & \multicolumn{2}{|c|}{70} \\
\hline Mean bias ( $\%$ of actual) & \multicolumn{2}{|c|}{7.1} & \multicolumn{2}{|c|}{7.3} \\
\hline SD of bias ( $\%$ of actual) & \multicolumn{2}{|c|}{9.4} & \multicolumn{2}{|c|}{4.5} \\
\hline
\end{tabular}

of the blood flow $\left(70 \times \sin 50^{\circ}=53 \mathrm{~cm} / \mathrm{s}\right)$. Fig. 9 shows the results from phantom experiments, where the A-line increment ranges from one-half to one lateral beam width as a result of the limitation of the Antares ultrasound imaging system. Again, it can be seen that the Q-factor of the power spectrum is high when the scan velocity $(52 \mathrm{~cm} / \mathrm{s})$ is close to the lateral component of the blood flow $\left(65 \times \sin 50^{\circ}=50 \mathrm{~cm} / \mathrm{s}\right)$. However, aliasing occurs because the blood flow was not adequately sampled (the maximum lateral spatial frequency of the speckle pattern is greater than the Nyquist frequency). These results indicate another potential method for 2-D blood flow velocity estimation. A high-lateral-resolution ultrasound imaging system may be used for data acquisition in future studies, which will eliminate aliasing for spectrum analysis. However, this would come at the cost of an increased number of A-lines, thereby slowing the frame rate.

Finally, some commentary on the length of time required to acquire data for flow estimates is appropriate here. The typical time for collecting one frame of data is around $40 \mathrm{~ms}$. Because nine frames were used for each estimation, the time for the first estimation would be around $360 \mathrm{~ms}$. However, subsequent estimates can overlap with previous frames (a rolling data collection), so subsequent estimates can be made in $40 \mathrm{~ms}$. Thus, about 25 samples/ heart cycle can be made using this algorithm, which is similar to color flow estimates in commercial machines.

\section{Conclusion}

This paper investigated a new algorithm for two-dimensional blood flow velocity estimation using the apparent speckle pattern angle. The apparent angle of speckle pattern changes with different scan velocities because of misregistration between the ultrasound beam and scatterers. When the scan velocity equals the lateral component of the blood flow, the apparent angle of the speckle pattern will equal the actual angle of blood flow and the aligned blood flow has a maximum speckle size because the speckle pattern is most coherent in this condition. Blood flow data from computer simulation and flow phantom experiments were both investigated. Our results showed that the 


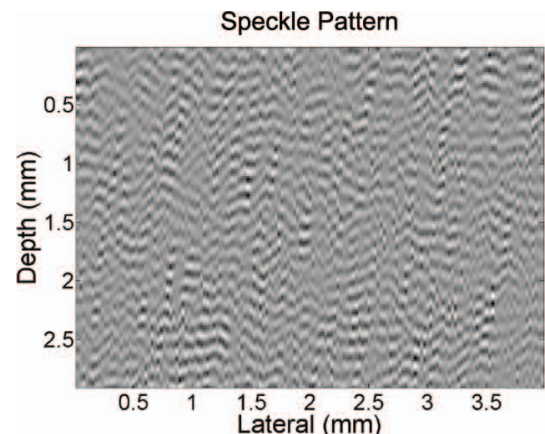

(a)

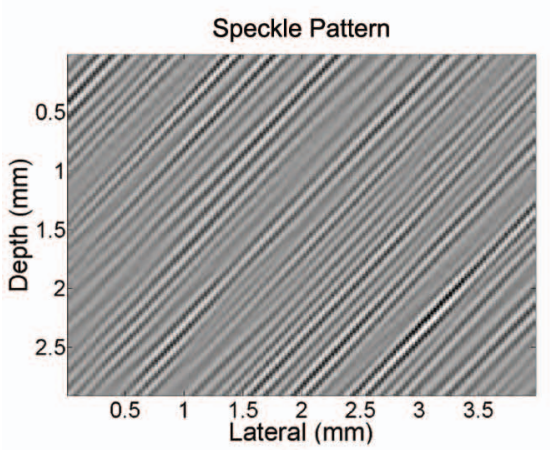

(b)

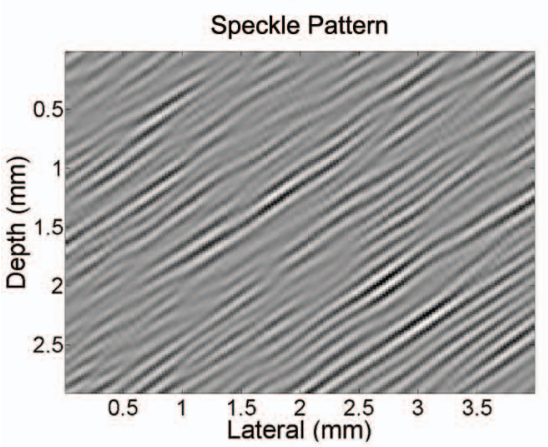

(c)

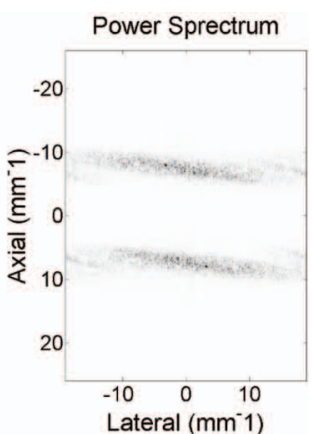

(d)

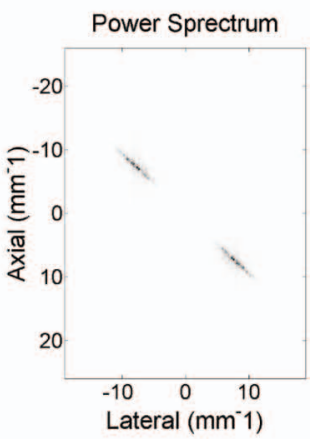

(e)

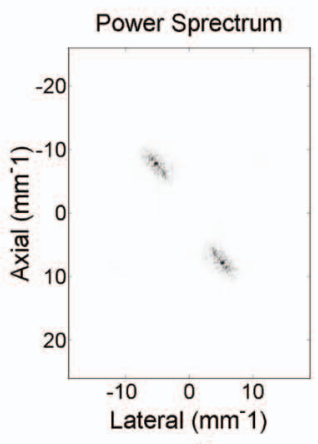

(f)

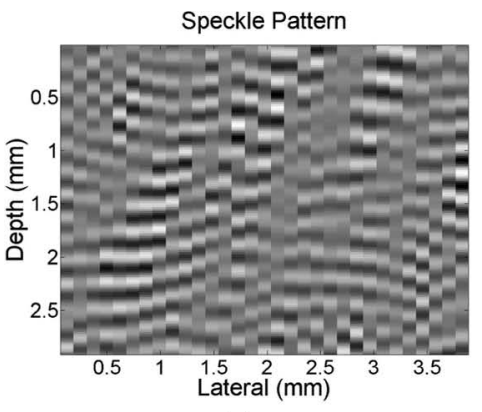

(a)

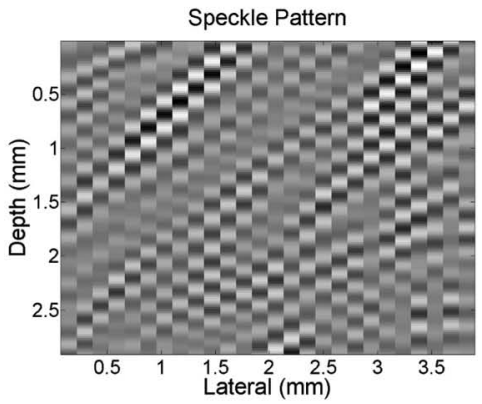

(b)

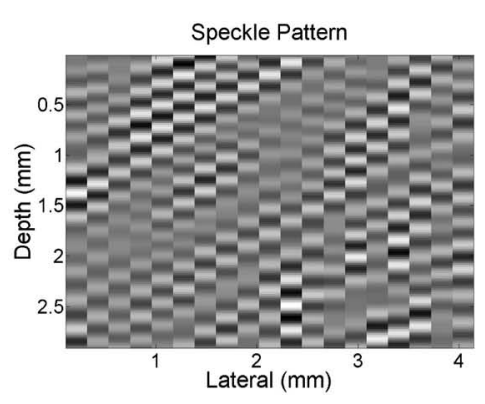

(c)

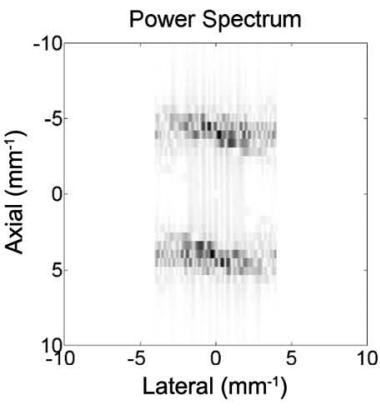

(d)

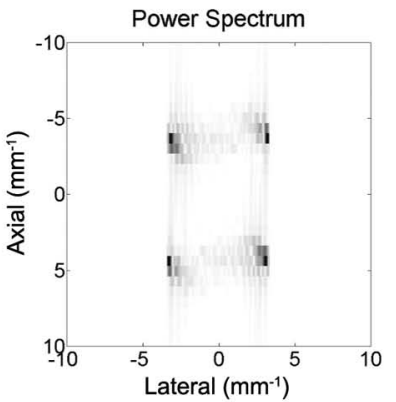

(e)

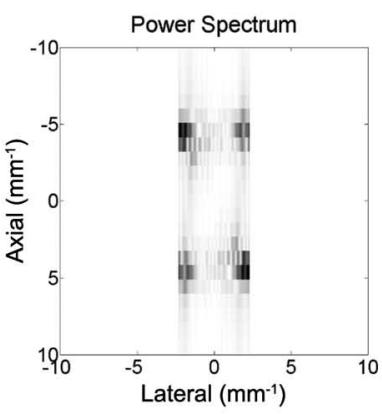

(f)

Fig. 9. The speckle pattern of the blood flow $\left(65 \mathrm{~cm} / \mathrm{s}, 50^{\circ}\right.$ relative to the ultrasound beam) collected by different scan velocities: scan velocity is (a) $22 \mathrm{~cm} / \mathrm{s}$, (b) $52 \mathrm{~cm} / \mathrm{s}$, and (c) $75 \mathrm{~cm} / \mathrm{s}$. The power spectrum of the speckle pattern: scan velocity is (d) $22 \mathrm{~cm} / \mathrm{s}$, (e) $52 \mathrm{~cm} / \mathrm{s}$, and (f) $75 \mathrm{~cm} / \mathrm{s}$. Blood flow phantom with A-line increment equal to one half of the lateral beam width [(a), (b), (d), and (e)], and one lateral beam width $[(\mathrm{c})$ and $(\mathrm{f})]$.

\section{REFERENCES}

proposed algorithm can resolve the amplitude and angle of the blood flow simultaneously. The angle and velocity estimation bias is below $5 \%$ in the simulated plug flow. Because of speckle decorrelation in simulated parabolic flow and the blood flow phantom, the angle of the blood flow will be overestimated and the velocity will be underestimated. The angle and velocity estimation bias increases with decreasing angle between blood flow and the ultrasound beam axis. This paper introduced a new algorithm for two-dimensional blood flow velocity estimation. Future proposed work includes using more scan velocities, determining realistic hardware and software requirements, and algorithm optimization, including the effects of optimal ROI selection and the signal-to-noise ratio.

[1] J. A. Jensen, Estimation of Blood Velocities using Ultrasound: A Signal Processing Approach. Cambridge, UK: Cambridge University Press, 1996.

[2] C. Kasai, K. Namekawa, A. Koyano, and R. Omoto, "Real-time twodimensional blood flow imaging using an autocorrelation technique," IEEE Trans. Sonics Ultrason., vol. 32, no. 3, pp. 458-463, 1985.

[3] I. A. Hein and W. D. O'Brien Jr., "Current time-domain methods for assessing tissue motion by analysis form reflected ultrasound echo-A review," IEEE Trans. Ultrason. Ferroelectr. Freq. Control, vol. 40, no. 2, pp. 84-102, 1993.

[4] A. Swillens, P. Segers, and L. Lovstakken, "A combined speckle tracking and phase-shift estimation approach for 2D blood flow imaging in the carotid bifurcation," in Proc. IEEE Ultrasonics Symp., 2010, pp. 1072-1075.

[5] M. D. Fox, "Multiple crossed-beam ultrasound Doppler velocimetry," IEEE Trans. Sonics Ultrason., vol. SU-25, no. 5, pp. 281-286, 1978 . 
[6] J. R. Overbeck, K. W. Beach, and D. E. Strandness, "Vector Doppler: Accurate measurement of blood velocity in two dimensions," Ultrasound Med. Biol., vol. 18, no. 1, pp. 19-31, 1992.

[7] M. Scabia, M. Calzolai, L. Capineri, L. Masotti, and A. Fort, "A real-time two-dimensional pulsed-wave Doppler system," Ultrasound Med. Biol., vol. 26, no. 1, pp. 121-131, 2000.

[8] B. Dunmire, K. W. Beach, K. Labs, M. Plett, and D. E. Strandness, "Cross-beam vector Doppler ultrasound for angle-independent velocity measurements," Ultrasound Med. Biol., vol. 26, pp. 1213-1235, Oct. 2000.

[9] G. E. Trahey, J. W. Allison, and O. T. Von Ramm, "Angle independent ultrasonic detection of blood flow," IEEE Trans. Biomed. Eng., vol. BME-34, no. 12, pp. 965-967, 1987.

[10] L. N. Bohs and G. E. Trahey, "A novel method for angle independent ultrasonic imaging of blood flow and tissue motion," IEEE Trans. Biomed. Eng., vol. 38, no. 3, pp. 280-286, 1991.

[11] G. R. Bashford and O. T. Von Ramm, "Ultrasound three-dimensional velocity measurements by feature tracking," IEEE Trans. Ultrason. Ferroelectr. Freq. Control, vol. 43, no. 3, pp. 376-384, 1996.

[12] J. Kuo and O. T. Von Ramm, "Three-dimensional motion measurement using feature tracking," IEEE Trans. Ultrason. Ferroelectr. Freq. Control, vol. 55, no. 4, pp. 800-810, 2008.

[13] M. E. Aderson, "Multi-dimension velocity estimation with ultrasound using spatial quadrature," IEEE Trans. Ultrason. Ferroelectr. Freq. Control, vol. 45, no. 3, pp. 852-861, 1998.

[14] J. A. Jensen and P. Munk, "A new method for estimation of velocity vectors," IEEE Trans. Ultrason. Ferroelectr. Freq. Control, vol. 45, no. 3, pp. 837-851, 1998.

[15] N. Oddershede, L. Lovstakken, H. Torp, and J. A. Jensen, "Estimating $2 \mathrm{D}$ vector velocities using multidimensional spectrum analysis," IEEE Trans. Ultrason. Ferroelectr. Freq. Control, vol. 55, no. 8, pp. 1744-1754, 2008.

[16] M. Schlaikjer and J. A. Jensen, "Maximum likelihood blood velocity estimator incorporating properties of flow physics," IEEE Trans. Ultrason. Ferroelectr. Freq. Control, vol. 51, no. 1, pp. 80-92, 2004.

[17] K. Shung, Diagnostic Ultrasound: Imaging and Blood Flow Measurements. Boca Raton, FL: CRC Press, 2005, pp. 72-73.

[18] M. F. Insana, "Ultrasonic imaging," in Wiley Encyclopedia of Biomedical Engineering, M. Akay, Ed., Hoboken, NJ: Wiley, 2006, pp. 3640-3648.

[19] T. Xu and G. R. Bashford, "Lateral blood flow velocity estimation based on ultrasound speckle size change with scan velocity," IEEE Trans. Ultrason. Ferroelectr. Freq. Control, vol. 57, no. 12, pp. 2695-2703, 2010.

[20] T. Xu and G. R. Bashford, "Resolving the lateral component of blood flow velocity based on ultrasound speckle size change with scan direction and speed," in Proc. Annu. Int. Conf. IEEE Engineering in Medicine and Biology Society, 2009, vol. 3-6, pp. 491-494.

[21] T. Xu and G. R. Bashford, "Further progress on lateral flow estimation using speckle size variation," in Proc. IEEE Ultrasonics Symp., 2009, pp. 1383-1386.

[22] J. A. Jensen and N. B. Svendsen, "Calculation of pressure fields from arbitrary shaped, apodized, and excited ultrasound transducers," IEEE Trans. Ultrason. Ferroelectr. Freq. Control, vol. 39, no. 2, pp. 262-267, 1992.

[23] J. A. Jensen, "Field: A program for simulating ultrasound systems," Med. Biol. Eng. Comput., vol. 34, suppl. 1, pt. 1, pp. 351-353, 1996.

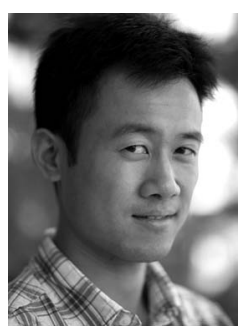

Tiantian Xu received the B.S. degree in biomedical engineering from the Huazhong University of Science and Technology, Wuhan, Hubei, P.R. China, in 2007 and the Ph.D. degree in biomedical engineering from the University of Nebraska, Lincoln, NE, in 2012. He was previously an Associate Ultrasound Scientist Consultant at Kona Medical Inc., Bellevue, WA. He is currently a Systems Engineer at GE Healthcare, Wauwatosa, WI. His research centers on new methods of ultrasound blood flow estimation, including feature tracking, speckle size estimation, and speckle pattern analyses.

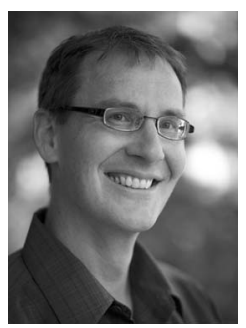

Gregory R. Bashford (M'96-SM'03) received the B.S. degree in electrical engineering from the University of Nebraska, Lincoln, NE and the Ph.D. degree in biomedical engineering from Duke University, Durham, NC. He was previously an Image Analysis Engineer at Acuson Corporation, Mountain View, CA; Systems Engineer at GE Medical Systems, Milwaukee, WI; and Senior Scientist at LI-COR Biosciences, Lincoln, NE. In 2003, he joined the faculty of the Biological Systems Engineering Department at the University of Nebraska. His research interests include methods and applications of biological and biomedical signal and image analyses. 